\title{
Research on the Effect Factor of WSN Nodes Ranging Using WTR Method in NLOS Environment
}

\author{
Zheng Zhang \\ School of Mechanical Engineering \\ Hubei University of Technology \\ Wuhan,China \\ email: zhangzheng_hb@sina.com \\ Chan wang \\ School of Mechanical Engineering, \\ Hubei University of Technology, \\ Wuhan,China, \\ email:1042875021@qq.com
}

\author{
X.P Tao \\ School of Mechanical Engineering, \\ Hubei University of Technology, \\ Wuhan,China, \\ email:185618351@qq.com \\ Lun Zeng \\ School of Mechanical Engineering, \\ Hubei University of Technology, \\ Wuhan,China, \\ email:zenglun2007@126.com
}

\begin{abstract}
Time reversal technique as an excellent channel equalization technology which can restrain multipath effectively to implementation time focusing and can improve the distribution of energy in space to implementation spatial focusing. This paper aim to improve the shortcomings with poor accuracy of positioning, a large amount of calculation,sensitive to the environment for the existing location method indoor.what make full use of strongly time resolution and spatiotemporal focusing characteristics of TR in UWB.From the perspective of collection multipath energy, which brings together time reversal theory, $U W B$ technology TOA to research the effect factor of obstacles in NLOS environment.This paper introduce ranging process of windowed time reversal in indoor environment. With a large number of ranging experiment are carried out,analysed the sources of measurement error in NLOS environment and compared its effects. Simulation results show that the influence of obstacles on ranging. From large to small in turn as the length,width and position which lay the foundation for the next positon.
\end{abstract}

Keywords-time reversal;spatiotemporal focusing;UWB;TOA; windowed time reversal.

\section{INTRODUCTION}

The federal communications commission (FCC) published the E-911 positioning service needs which request all wireless operators have to provide mobile location services for call 911 emergency calls [1] before October 2001, and meet the accuracy of positioning what the probability no less than $67 \%$ within $125 \mathrm{~m}$, the probability no less than $95 \%$ within $300 \mathrm{~m}$. So the wireless position is becoming a hot spot in recent research.The measurement value of arrival time or time difference will appear excessive time delay, when can only spread in nonline-of-sight manner between the waves and cell base station.Namely, the NLOS error.The field measurement result of Nokia company shown that the NLOS error reach 500-700m in the GSM network environment.Korea Telecom's measurement result show that the NLOS error attain $589 \mathrm{~m}$ in the IS-95 network.Traditional positioning methods,such as Chan[2],Fang[3],Taylor series expansion algorithm[4] and so on which without considering the influence of the NLOS error.So the positioning accuracy of these methods will be greatly reduced.it is also not up to E-911 requirement of FCC.So researching on positioning method of eliminate NLOS error was widespread attention. Use $\mathrm{N}$ polynomial for smoothness to TDOA measurement data in a period of time, according to the offset between smooth value and measured value which use for correction of smooth curve[5]; The weighted average with positioning results in in order to reduce the NLOS error[6].Using Calman filtering technology to realize mobile tracking and mitigate the influence of NLOS error[7-8].Time reversal technique originated in the time reversal mirror,and time reversal mirror originated in the phase conjugation technology of optical Which was first used in the field of ultrasonic application by M.Fink in 1992[9].Using time reversal technology can play a balanced role on nonhomogeneous,realize synchronous focusing of acoustic wave in time and space.Then,scholars launched application study in various fields.In medicine,time reversal can be used for the detection of breast cancer,the treatment of stone[10].In the military,it can be used to realize underwater communication and command. With the in-depth research of time reversal technology,people found that the technology is not only suitable for optical and ultrasonic field,but also can realize spatiotemporal focusing of electromagnetic wave in the field of electromagnetic wave propagation[11].After that,people in microwave imagine[12-13], wireless communication[1 4-16], antenna array[17],cancer treatment and other fields which has made a series of achievements. This paper put forward the effect factors of measurement error in NLOS environment. 


\section{THE TIME REVERSAL TECHNIQUE}

Time reversal technique is based on the principle of superposition and the principle of reciprocity. Through to the actual channel impulse response in the time domain processing in reverse order, what is realizing the perfect restoration of signals in location node finally.Time reversal technique can exploit the multipath effect which produced in communication process to realize the perfect restoration of electromagnetic signal and spatiotemporal focusing.This paper use multipath characteristics and high resolution of UWB signals, spatiotemporal focusing properties of time reversal to research the effect factor of obstacles. The TR technology described below is how to achieve the rehabiltation and spatiotemporal focusing Properties of electromagnetic signals.

\section{A. Temporal focusing properties of time reversal}

The channel impulse response (channel impulse response, CIR) is an important concept study on wireless propagation of TR electromagnetic wave. If the channel response $\mathrm{h}(\mathrm{t})$ between the two ordinary sensor nodes can be expressed as

$$
h(\mathrm{t})=\sum_{i=1}^{N} a_{i} \delta\left(\mathrm{t}-\tau_{i}\right)
$$

where $\delta(\mathrm{t})$ is the Dirac Delta function.represent the ith path channel gain and time delay at the time of $t$, respectively. $\mathrm{N}$ is the number of multipath. When the launch signal is $\mathrm{x}(\mathrm{t})$, the received signal $\mathrm{y}(\mathrm{t})$ can be shown as

$$
y(\mathrm{t})=x(\mathrm{t}) * h(\mathrm{t})=x(\mathrm{t}) * \sum_{i=1}^{N} a_{i} \delta\left(\mathrm{t}-\tau_{i}\right)
$$

After reversal of $y(t)$ in the domain at the reference node, we can get

$$
y(-\mathrm{t})=x(-\mathrm{t}) * h(-\mathrm{t})
$$

In the second wave propagation,y(-t) is back propagated by the reference node to the position node, then the focusing signal received by the position node can be given as

$$
\begin{aligned}
y_{T R}(-\mathrm{t}) & =y(-\mathrm{t}) * h(\mathrm{t}) \\
& =x(-\mathrm{t}) * h(-\mathrm{t}) * h(\mathrm{t}) \\
& =x(-\mathrm{t}) * h_{e q}(\mathrm{t})
\end{aligned}
$$

According to the form of TR communication system, in addition to pulse signal which can be unified equivalent channel component, the equivalent channel is $h_{e q}(\mathrm{t})=h(-\mathrm{t}) * h(\mathrm{t})$

$=\left(\sum_{i=1}^{N} a_{i} \delta\left(-\mathrm{t}-\tau_{i}\right)\right) *\left(\sum_{j=1}^{N} a_{j} \delta\left(\mathrm{t}-\tau_{j}\right)\right)$

$=\sum_{i=j=1}^{N} a_{i}^{2} \delta\left(-\mathrm{t}-\tau_{i}\right) * \delta\left(\mathrm{t}-\tau_{i}\right)+\sum_{i=1}^{N} \sum_{j=1, i \neq j}^{N} a_{i} a_{j} \delta\left(-\mathrm{t}-\tau_{i}\right)$

$* \delta\left(\mathrm{t}-\tau_{j}\right)$

$=\sum_{i=j=1}^{N} a_{i}^{2} \delta(\mathrm{t})+\sum_{i=1}^{N} \sum_{j=1 \cup j \neq i}^{N} a_{i} a_{j} \delta\left(\mathrm{t}+\tau_{\mathrm{i}}-\tau_{\mathrm{j}}\right)$
From(2.5),it is obvious that the first of equivalent channel which is the autocorrelation function of the channel impulse response.It show that their original multipath signals collected on the focus at the same time and formed a "impulse"after the coherent superposition. when $\mathrm{t}=0$,it is easily known that $\mathrm{heq}_{\mathrm{e}}(0)=\sum_{i=1}^{N} a_{i}^{2}$ is the maximum value of the time reversaloperator.Namely,

$h_{e q}(0)$ is the operator main lobe. When $t=t_{n}$, it represent the nth side lode of the time reversal operator.Suppose that there are rich multipath environment,side lobe will approximate to zero due to incoherent superposition with each other which illustrates the focusing on characteristic of time reversal.

\section{B. Spatial focusing properties of time reversal}

The channel impulse response in addition to be able to describe temporal focusing properties accurately, but it can to characterize spatial focusing properties of time reversal.The time reversal focusing process can be considered as relevant result of time reversal impulse response $h\left(-t_{a b}\right)$ and actual channel impulse response $h\left(t_{a b}\right)$ which produce between position nodes and reference nodes(i.e a,b).Using the relevant principle,you can calculate the received energy values at other nontarget node c:

$$
E_{a c}=x(t) * h\left(-t_{a b}\right) * h\left(t_{a b}\right)
$$

Where $x(t)$ is transmitting signals for node a, $E_{a c}$ is the observed signal energy at non-target node c.Under the condition of the energy of transmit signal $x(t)$ constant, the energy is mainly depend on the relevance degree of time reversal impulse response between node $\mathrm{a}$ and $\mathrm{b}$ and actual channel impulse response between node a and c.Due to the space position is different of target node $b$ and non-target node c,the channel impulse response is also different between $a b$ and ac.The farther distance between them,the weaker correlation they have.The nontarget node c receives less signal energy from node a,which can present a spatiotemporal properties of electromagnetic signals.

\section{INDOOR NODES DISTANCE MEASUREMENT IN NLOS}

In the NLOS environment, the reflection or blocking of electromagnetic wave by the obstacles will cause received wave distortion and peak drift.The conventional method to measure the TOA by detecting the received wave's peak causes large error.The receive signal contains the response of original test signal.So,this paper use the maximum-likelihood method.Finding the signal which is similar to the original test signal in the receive signal and beginning to window time reversal.

\section{A. Nodes ranging process}

The principle of this method can be described as the following steps. As shown in Fig .1. 


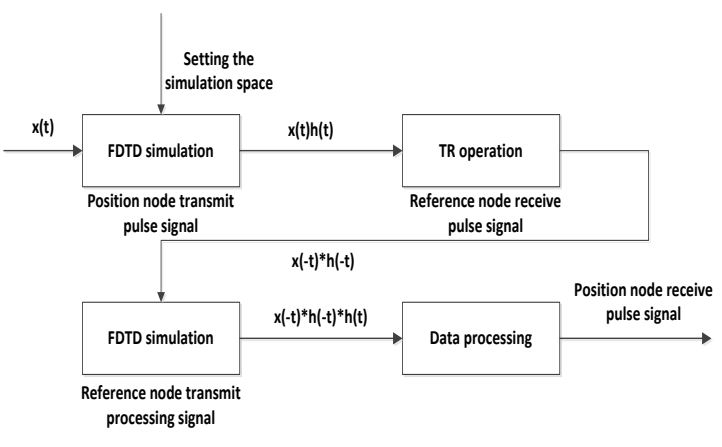

Figure 1. Ranging process of time reversal

- Position node transmit a test signal

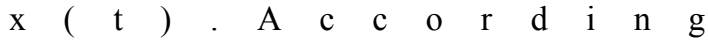
to the characteristic of the time reversal operator. To achieve the effect of focusing,the test pulse must be used even function.In complex environment,in order to better adapt to the multipath environment and good anti-interference capability, the second orderGaussian pulse is used as the probe signal in this paper.

- After multipath effects,the reference node received test signal which came from the position node.Use the maximum-likelihood method to find out the signal which is similar to the original test signal and the same width in the receive signal,then begin to window time reversal.

- After time reversal of windowed signal,it can obtain multipath inversion pulse $x(t) * h(-t)$ then transmit it to position node.

- Position node received the inversion signal after spatiotemporal focusing. The main lobe of pulse waveform is an approximate axial symmetry pulse and it is is similar to the second derivative function of Gaussian pulses.

- $\quad$ Calculating the distance between two nodes, then get the measurement results.

\section{$B$. The analysis and improvement of ranging factors under NLOS environments}

In the NLOS environment,electromagnetic pulse is reflected or blocked by obstacles, it will cause time delay when arrived,it also will produce large errors in calculation. This paper mainly studies the obstacles which lie in line-of-sight, and it will cause NLOS in the twodimensional environment. The main effect factors for ranging include:a)the position of obstacles; $b$ )the length of obstacles;c)the width of obstacles.In this paper,we use Matlab to build a visual simulation platform which is based on the finite difference time domain(FDTD) method.In the visual simulation space, there is only one position node $(0.5,1.3)$ and reference node $(2,1.3)$.Adding obstacles between two nodes.By changing the effect factor of obstacles to research the influence on ranging precision. Distribution of the simulation space shown in Fig .2:

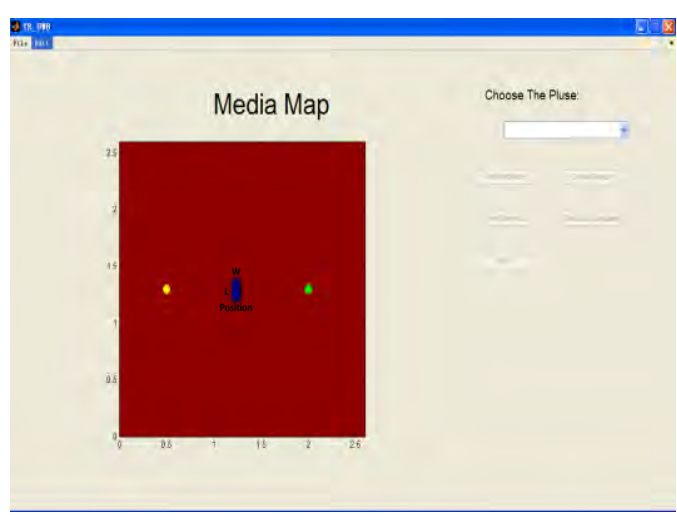

Figure 2. The distribution of simulation space

First, position node transmit signal $\mathrm{x}(\mathrm{t})$ to reference node.The received signal of reference node is $y(\mathrm{t})=x(\mathrm{t}) * h(\mathrm{t})=\sum_{i=1}^{N} a_{i} \delta\left(\mathrm{t}-\tau_{i}\right)$.

The electromagnetic wave peak is $\mathrm{P}_{\mathrm{N}}=\sum_{i=1}^{N} \mathrm{a}_{\mathrm{i}}$.then it will inverse the received signal and obtain focused energy of electromagnetic waves is $E_{N}=\sum_{i=1}^{N} a_{i}^{2}$.We will be evaluated them to find out factor which influence focusing effect, and propose improvement program.

1) The impact of obstacle's position on ranging

When the position of obstacle as only variable, Changing position which means moving obstacles in line-of-sight.That will have an effect on ranging.we start to measurement at a distance of $100 \mathrm{~mm}$ in line-of-sight which range from position node and move small obstacles (section dimension is $200 * 100$ ) every $100 \mathrm{~mm}$ distance. Then measured 13 groups data, That we can get the maximum focused energy value is E7. There are 13 groups focused energy value were calculated with E7 normalization.It will get the normalization curve of focused energy value and peak value which shown in Fig .3:

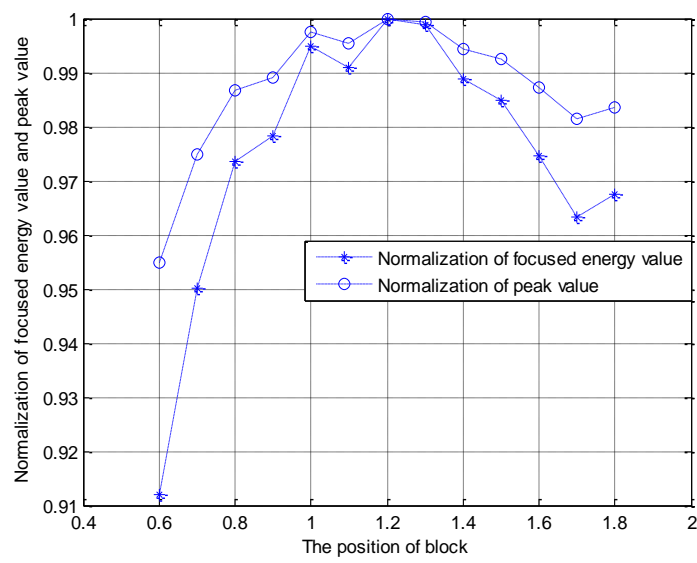

Figure 3. The position of obstacle 
The simulation results show that when the obstacle lie in symmetrical location of position node and reference node in line-of-sight, it has similar effect on channel.And the energy loss is the lowest in the central location, It is also say that it has the least impact on ranging.From $0.6 \mathrm{~m}$ to the center position at $1.2 \mathrm{~m}$ and then to $1.8 \mathrm{~m}$,normalization curve of focused energy value and peak value which use $1.2 \mathrm{~m}$ straight line as the center axis,it form downward opening parabola.

2) The impact of obstacle's length on ranging

When the length of obstacle as only variable,The size of length which impact on the ranging precision.Placing the obstacle $(100 * 100)$ in the center position between nodes in line-of-sight.Extended length every $50 \mathrm{~mm}$ on both sides along with length direction(total increment is 100 m).Measured 6 groups focused energy value,that we can get the maximum focused energy value is E1. There are 6groups focused energy value were calculated with E1 normalization.It will get the normalization curve of focused energy value and peak value which shown in Fig .4:

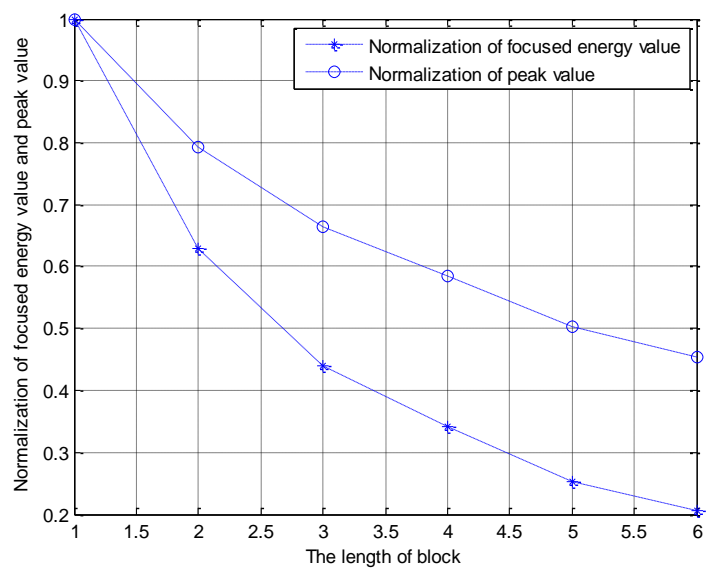

Figure 4. The length of obstacle

The simulation result show that the length of obstacles have a great effect on the value of focused energy value and peak value.In other words, the focused energy value and peak value decrease sharply.But the change rate of received value decreases gradually and tend to smooth. Namely the effect on ranging accuracy tend to be decreasing.

3) The impact of obstacle' $s$ width on ranging

When the width of obstacle as only variable.The size of width which impact on the ranging precision.Placing the obstacle $(100 * 100)$ in the center position between nodes in line-of-sight.Extended width every $50 \mathrm{~mm}$ on both sides along with width direction(total increment is $100 \mathrm{~mm})$. Measured 6 groups focused energy value,that we can get the maximum focused energy value is E1. There are 6 groups focused energy value were calculated with E1 normalization.It will get the normalization curve of focused energy value and peak value which shown in Fig .5:

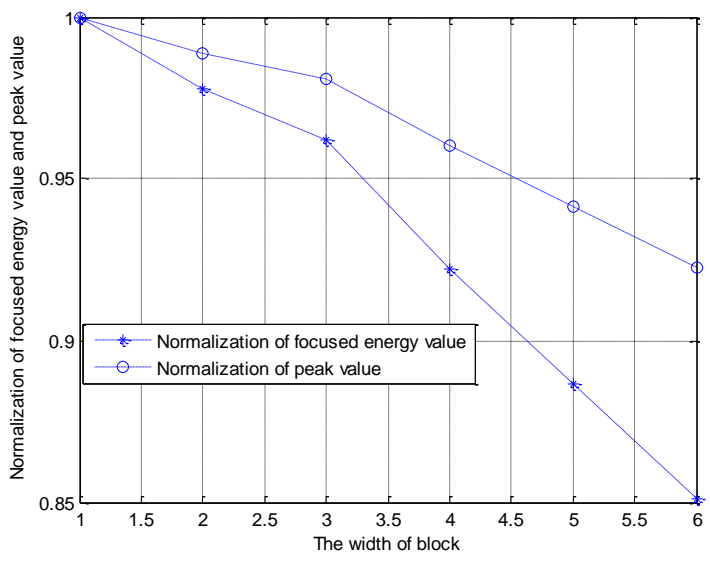

Figure 5. The width of obstacle

The simulation result show that compared with length, the effect on width is slight. But with the width increased, the focused energy value and peak value decrease gradually.And with increased width of obstacle, the change rate will increase gradually. That is the effect on ranging accuracy increasing.

\section{CONCLUSION}

This paper introduce the applications and spatiotemporal focusing property of time reversal techniques.It is also narrate the process of distance measurement method which based on time reversal and emphatically study the effect of raging which cause by obstacles position,length,width. According to a large number of experiments, we can know that the maximum influence is length.

\section{ACKNOWLEDGMENT}

This work was supported by the National Nature Science Foundation of China(Grant no. NFSC\#51174084 and Grant no.NFSC\#51121005), the National Nature Science Foundation of Hubei Province(Grant no.2010CD B02504), the Foreign Scientific and Technological Cooperation Project of the Science and Technology Department of Hubei Province(Grant no.2012HA00401), and Backbone Teachers Research Abroad Program of HuBei University of Technology.

\section{REFERENCES}

[1] REED J,RAPPAPORT T.An overview of the challenges and progress in meeting the E-911requirement for location Service[J].IEEE Communic ation Magazine 1998,36(4):30-37.

[2] Chan Y. and Ho K.A simple and efficient estimate for hyperbolic location[J].IEEE Transactions on Signal Processing,1994,42(8):1905- 1915.

[3] FANG B T.Simple solutions for hyperbolic and related position fixes [J].IEEE Transactions on Aerospace and Electronic Systems 1990, 26(9):748-753.

[4] Foy W.Position-location solutions by Taylor series estimation[J].IEEE Transactions and Electronic Systems,1976,12(3):187-193.

[5] WYLIEM P, HOLTZMAN J.The non-line-of-sight problem in mobile location estimate[A].IEEE international conference on universal personalcommunicat ion[C].1996.827-831.

[6] CHEN Pichun.A non-line-of-sight error mitigation algorithm[A]. IEEE Wireless Comm unication and Networking Conference (WCNC99)[C].New Orleans USA,1999.1(9):316-320 
[7] Le B.L.,Ahmed K.,Tsuji H.Mobile location estimator with NLOS mitigation using kalman filtering[C].WCNC2003,New Orleans,USA, 2003:1969-1973.

[8] Liao J.F., and Chen B.S. Robust mobile location estimator with NLOS Mitigation using interacting multiple model algorithm[J].IEEE Transactions on Wireless Communication ,2006,5(11):3002-3006.

[9] M Fink.Time reversal of ultrasonic fields-part I:basic principles.IEEE Trans on Ultrasonics,Ferroelectronics and Frequency Control,1992, 39(5):555-566.

[10] Francois Wu,Jean-Louis Thomas.Time reversal of ultrasonic fields -part II:Experimental Results.IEEE Trans on Ultrasonics, Ferroelectrics and Frequency Control,Sep.1992,39(5):567-578.

[11] G. Lerosey,J.de Rosny.Time reversal of wideband microwave. Applied Physics Letters,2006,88(15):154101-154103.

[12] Moura.J.M.F,Yuanwei Jin.Time Reversal Imagine by Adaptive Interference Canceling.IEEE Transactions on Signal Processing,2008, 56(1):233-247.
[13] [Dehong Liu,Gang Kang,Ling Li,Ye Chen,Vasudevan,S.Joines,W, Qing Huo Liu,Krolik.J,Carin.L.Electromagnetic time-reversa imagine of a Target in a cluttered environment.IEEE Transactions on Antennas and Propagation,2005,53(9):3058-3066.

[14] Qiu R C,Zhou C,Guo N,and Zhang J Q.Time reversal with MISO for ultra-Wideband communications: experimental results.IEEE Antenna and Wireless Propagation Letters,2006,5(2):269-273.

[15] R.C.Qiu,C.Zhou,N.Guo.Time reversal with MISO for ultraWideband Communications:experimental results.IEEE Radio and Wireless Symposium,2006,5(6):499-502.

[16] P.Kyritsi,G Papanicolaou ,P.Eggers, etal.MISO time reversal and delay-spread compression for FWA channels at 5GHZ.IEEE Antennas and Wireless Propagation Letters,2004,3(1):96-99.

[17] H.T.Nguyen,J.B. Andersen and G.F.Pedersen. The potential use of time reversal technique in multiple elements antenna systems. Communications 